\section{Efficient Test Access Mechanism Optimization for System-on-Chip}

Vikram Iyengar, Krishnendu Chakrabarty, and Erik Jan Marinissen

\begin{abstract}
Test access mechanisms (TAMs) are an important component of a system-on-chip (SOC) test architecture. TAM optimization is necessary to minimize the SOC testing time. We present a fast, heuristic technique for TAM optimization and demonstrate its scalability for several industrial SOCs. Since the TAM optimization problem is $\mathcal{N} \mathcal{P}$-hard, recently proposed methods based on integer linear programming and exhaustive enumeration can be used to design limited test architectures with only a very small number of TAMs in a reasonable amount of time. In this paper, we explore a larger solution-space to design efficient test architectures with more TAMs. We show that the SOC testing times obtained using the new heuristic algorithm are comparable to or lower than the testing times obtained using enumeration. Moreover, significant reduction can be obtained in the CPU time compared to enumeration.
\end{abstract}

Index Terms-Core-based systems, heuristic methods, system-on-chip, test access mechanism, testing time.

\section{INTRODUCTION}

Modular testing of embedded cores in a system-on-chip (SOC) is now widely advocated to simplify the complex problems of SOC test access and test application [9], [21], [23]. Modular test refers to the process of isolating embedded cores and groups of cores, and gaining test access to these separate SOC partitions for test data delivery. The primary motivation for modular test is to use a "divide-and-conquer" approach and to promote the re-use of precomputed test sets for embedded cores. In addition, for nonlogic cores such as memories, nonscan legacy cores, or hard-IP, core isolation and modular test are often the only available options for test.

Test data delivery to embedded cores for modular test is a challenging problem during system-level test integration. Dedicated core test wrappers and test access mechanisms (TAMs) are often used for plug-and-play SOC testing. Test wrappers are used to isolate the core, adapt the TAM width to the number of wrapper input-outputs (I/Os), and apply tests. TAMs transport test data between SOC pins and test wrappers [23]. The optimization of wrappers and TAMs in conjunction is, therefore, critical because the testing time for a core varies widely with the TAM width, the wrapper design, and the manner in which TAM wires are connected to wrapper scan chains.

Most prior research has either studied wrapper design and TAM optimization as independent problems [1], [4], [5], [18], or has not addressed the issue of sizing the TAMs to minimize SOC testing time [22]. Alternative approaches that combine TAM design with test scheduling [14], [15], [19] do not address the problem of wrapper design and its relationship to TAM optimization. Recent methods

Manuscript received June 14, 2002; revised September 23, 2002. This work was supported in part by the National Science Foundation under Grant CCR9875324 and Grant CCR-0204077. The work of V. Iyengar was supported by an IBM Graduate Fellowship. A preliminary version of this paper appeared in Proc. Design Automation and Test in Europe Conf., pp. 491-498, Mar. 2002. This paper was recommended by Associate Editor N. K. Jha.

V. Iyengar was with the Department of Electrical and Computer Engineering, Duke University, Durham, NC 27708 USA. He is now with IBM Microelectronics, Essex Junction, VT 05452 USA (e-mail: vikrami@us.ibm.com).

K. Chakrabarty is with the Department of Electrical and Computer Engineering, Duke University, Durham, NC 27708 USA (e-mail: krish@ee.duke.edu).

E. J. Marinissen is with Philips Research Laboratories, 5656 AA Eindhoven, The Netherlands (e-mail: Erik.Jan.Marinissen@philips.com).

Digital Object Identifier 10.1109/TCAD.2003.810737 include test resource allocation and scheduling using rectangle packing [8], graph formulation and dependency matrix partitioning [9], and bipartite graph matching [13]. The techniques proposed in [8] and [9] provide an efficient mapping of core I/Os to SOC pins for test scheduling.

In [10], four problems structured in order of increasing complexity were formulated, such that they serve as stepping stones to the problem of TAM optimization for SOCs. We first review these four problems.

1) $\mathcal{P}_{\mathrm{W}}$ : Design a wrapper for a given core, such that the core testing time is minimized, and the TAM width required for the core is minimized.

2) $\mathcal{P}_{\text {Aw }}$ : Determine i) an assignment of cores to TAMs of given widths and ii) a wrapper design for each core such that SOC testing time is minimized. (Item (ii) corresponds to $\mathcal{P}_{\mathrm{W}}$.)

3) $\mathcal{P}_{\text {PAw }}$ : Determine i) a partition of the total TAM width among the given number of TAMs, ii) an assignment of cores to the TAMs, and iii) a wrapper design for each core such that SOC testing time is minimized. (Items (ii) and (iii) together correspond to $\mathcal{P}_{\text {Aw }}$.)

These three problems lead up to $\mathcal{P}_{\mathrm{NPAW}}$, the more general problem, described as follows.

4) $\mathcal{P}_{\text {NPAW }}$ : Determine i) the number of TAMs for the SOC; ii) a partition of the total TAM width among the TAMs; iii) an assignment of cores to TAMs; and iv) a wrapper design for each core, such that SOC testing time is minimized. (Items (ii), (iii), and (iv) together correspond to $\mathcal{P}_{\text {PAw }}$.)

The above four problems are all $\mathcal{N} \mathcal{P}$-hard [10].

The first integrated method for jointly optimizing wrappers and TAMs was proposed in [10]. TAM optimization was carried out by enumerating over the number of TAMs on the SOC as well as over the different partitions of TAM width. Integer linear programming (ILP) was used to calculate the optimal core assignment and resulting testing time for each partition. A drawback of this approach is that a large solution-space cannot be searched rapidly enough and, therefore, the TAM designs considered in [10] are limited to only two or three TAMs in order to maintain feasible compute times. However, architectures having only two or three TAMs are not always effective because the testing time can often be reduced by increasing the number of TAMs. This is because when there are multiple TAMs of different widths, a larger number of cores can be assigned to TAMs whose widths match the cores's own test data requirements; thus, the number of unnecessary TAM wires assigned to cores is reduced. Secondly, multiple TAMs provide greater test parallelism and TAM wires deemed unnecessary for certain cores can be used to transport test data for other cores, thereby decreasing total testing time. The methods in [10] are, therefore, inadequate for large industrial SOCs.

In this paper, we present a heuristic approach to solve the TAM optimization problem for industrial SOCs. Compared to [10], the heuristic approach offers the following important advantages.

- We can search a larger solution-space than was possible earlier.

- We can design more effective test architectures having a larger number of TAMs.

- In several cases, we obtain lower testing times than were obtained earlier.

- We significantly reduce the computation time compared to [10].

As in [10], we assume the fixed-width test bus architecture for TAMs. At most one core can be tested per TAM at any time. However, the TAMs operate independently of each other. To solve $\mathcal{P}_{\mathbf{W}}$, we use the Design_wrapper algorithm presented in [10]. We then describe a new algorithm to solve problem $\mathcal{P}_{\text {AW }}$ efficiently. The solution to $\mathcal{P}_{\text {AW }}$, thus obtained, is a good approximation of the optimal 
solution for a given TAM width partition. An efficient technique for TAM partition enumeration is used to search a larger solution-space to obtain the number of TAMs and TAM width partition with the lowest testing time (problems $\mathcal{P}_{\text {PAW }}$ and $\mathcal{P}_{\text {NPAW }}$ ). This yields an intermediate solution to $\mathcal{P}_{\text {NPAW }}$. In the final step, an exact mathematical programming model is used to optimize the final assignment of cores and the SOC testing time. This three-step approach allows us to apply our methods to design effective test architectures having a larger number of TAMs for industrial SOCs. We show that while the SOC testing times obtained using the new heuristic algorithm are comparable to or lower than the testing times obtained using exact methods, a significant reduction is obtained in the CPU time needed. This is especially important since in some cases, the minimum SOC testing time is obtained for a larger number of TAMs, which we could not compute earlier in a reasonable amount of execution time.

We have not investigated core placement-and-layout issues, TAM routing constraints and effects on floorplanning in this paper. Place-and-route issues were addressed in [4], [11], [14], and [15]. However, constraints that limit core assignment to TAMs near the core can be added to our core assignment algorithm in the form of designer-specified preferences, as was presented in [4].

The organization of this paper is as follows. In Section II, we present a new heuristic algorithm to efficiently obtain an assignment of cores to TAMs of given widths (Problem $\mathcal{P}_{\text {AW }}$ ). In Section III, we describe how the new algorithm for $\mathcal{P}_{\text {AW }}$ is used to construct an algorithm for problems $\mathcal{P}_{\text {PAW }}$ and $\mathcal{P}_{\text {NPAW }}$ that identifies an effective number of TAMs and an effective partition of TAM widths. We also present the ILP model that is used for fine-grained optimization of the final core assignment. Finally, in Section IV, we present experimental results on four benchmark SOCs. A comparison of results with those presented in [10] demonstrates that near-optimal test architecture design with a larger number of TAMs can be obtained with significant reduction in CPU time.

\section{New Algorithm FOR CORE ASSIGNMENT}

The first problem $\mathcal{P}_{\mathbf{W}}$ is that of designing an optimal wrapper for a core. The $\mathcal{P}$ w problem has two priorities: i) minimizing core testing time and ii) minimizing the TAM width required for the test wrapper. To solve the problem, we use the Design_wrapper algorithm (proposed earlier in [10]) based on the best fit decreasing (BFD) heuristic for the bin packing problem [6]. The algorithm achieves priorities (i) and (ii) by balancing the lengths of the wrapper scan chains designed and identifying the number of wrapper scan chains that actually need to be created to minimize testing time. Priority (ii) is addressed by the algorithm since it has a built-in reluctance to create a new wrapper scan chain, while assigning core-internal scan chains and wrapper cells to the existing wrapper scan chains.

In this paper, we assume a fixed-width TAM architecture in which each TAM is independent of the other TAMs, however, the tests on each TAM are applied sequentially. TAM assignment to cores is, therefore, on a per-bus basis. Flexible-width TAM architectures can also be designed in which TAM assignment to cores is on a per-wire basis [9], [12]. A single flexible-width TAM can, therefore, test several cores simultaneously. However, the routing complexity is increased since TAM wires fork-and-merge between TAMs. Moreover, test conflicts can be created if two cores that must not be tested concurrently receive test patterns at the same time.

The second problem $\mathcal{P}_{\text {AW }}$ is that of assigning cores to TAMs of given widths. An ILP model was developed to solve $\mathcal{P}_{\text {AW }}$ exactly in [10]. The CPU time for this ILP model was reasonably short for a single execution and optimal solutions for the core assignment problem were easily obtained. However, $\mathcal{P}_{\text {AW }}$ is an $\mathcal{N} \mathcal{P}$-hard problem, and execu-

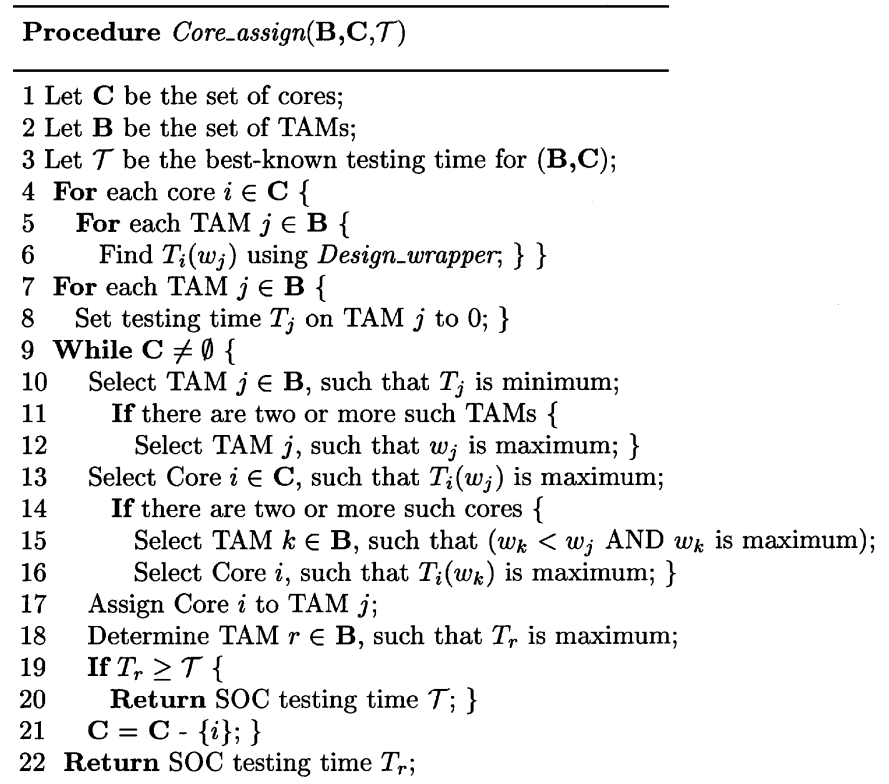

Fig. 1. New algorithm for core assignment.

tion times can get high for large problem instances. Furthermore, in [10], solutions to the problems $\mathcal{P}_{\text {PAW }}$ and $\mathcal{P}_{\text {NPAW }}$ were obtained by enumerating optimal ILP solutions to $\mathcal{P}_{\text {AW }}$ for each TAM width partition on the SOC. As a result, CPU times for $\mathcal{P}_{\text {PAW }}$ and $\mathcal{P}_{\text {NPAW }}$ were found to be prohibitively large for more than three TAMs. Moreover, during execution of the ILP model for $\mathcal{P}_{\text {AW }}$, the value of SOC testing time $\mathcal{T}$ is calculated only at the end of each iteration. Therefore, execution of the ILP model cannot be halted prematurely if it is discovered that the testing time of a TAM has already exceeded the best-known value $\mathcal{T}$ calculated earlier. Therefore, a faster algorithm for $\mathcal{P}_{\text {Aw }}$ that produces efficient results in polynomial time is clearly needed.

Here, we present a new heuristic algorithm for $\mathcal{P}_{\text {AW }}$ based on the relationship between TAM width and core testing times calculated for $\mathcal{P}_{\mathbf{W}}$. We design the heuristic for $\mathcal{P}_{\mathrm{AW}}$, based on an approximation algorithm for the problem of scheduling $n$ independent jobs (tests) on $m$ parallel, equal processors (TAMs) [3]. We extend the algorithm in [3] to address the case of scheduling jobs on unequal processors. The pseudocode for our heuristic Core_assign is presented in Fig. 1. In each iteration, the algorithm calculates the summed testing time on each TAM by adding up the testing times of all the cores assigned to that TAM. Then, the core with the largest testing time (among all unassigned cores) is assigned to the TAM with the shortest current summed testing time. Furthermore, during core assignment, if the time $T_{r}$ on any TAM $r$ exceeds the best-known value $\mathcal{T}$ computed in an earlier iteration and input to the algorithm in the current iteration, the algorithm returns $\mathcal{T}$ and halts. This plays a significant role in reducing the amount of computation when Core_assign is executed a large number of times, as will be shown in Section III.

We illustrate the Core_assign algorithm using an example SOC containing five cores and three TAMs. The testing times for the five cores $A$ to $E$ when assigned to the TAMs of widths 8,16 , and 32 are shown in Fig. 2(a). Initially, in lines 10-12, the testing time on all TAMs is zero cycles; therefore TAM 1 of width 32 , being the widest, is considered first. Core $E$ has the highest testing time on TAM 1, therefore, Core $E$ is assigned to TAM 1 (line 13). Next, there is a choice between Cores $A$ and $C$ to be assigned to TAM 2 of width 16 . We choose to assign Core $A$ to TAM 2 here because the testing time for Core $A$ on TAM 3 is higher than the testing time for Core $C$ on TAM 3 (lines 14-16 of Core_assign). This "look-ahead" heuristic assigns Core $A$ to TAM 2 rather than 


\begin{tabular}{|c||c|c|c|}
\hline \multicolumn{1}{|c||}{} & \multicolumn{3}{c|}{ Testing time (cycles) } \\
\cline { 2 - 4 } Cores & $\begin{array}{c}\text { TAM 1 } \\
\text { 32 bits }\end{array}$ & $\begin{array}{c}\text { TAM 2 } \\
16 \text { bits }\end{array}$ & $\begin{array}{c}\text { TAM 3 } \\
8 \text { bits }\end{array}$ \\
\hline \hline$A$ & 50 & 100 & 200 \\
\hline$B$ & 75 & 95 & 200 \\
\hline$C$ & 90 & 100 & 150 \\
\hline$D$ & 60 & 75 & 80 \\
\hline$E$ & 120 & 120 & 125 \\
\hline
\end{tabular}

(a)

\begin{tabular}{|c||c|c|}
\hline Core & TAM & $\begin{array}{c}\text { Testing } \\
\text { time } \\
\text { (cycles) }\end{array}$ \\
\hline \hline$A$ & 2 & 100 \\
$B$ & 3 & 200 \\
$C$ & 2 & 100 \\
$D$ & 1 & 60 \\
$E$ & 1 & 120 \\
\hline
\end{tabular}

(b)

Fig. 2. Core testing times for (a) the SOC used to illustrate Core_assign and (b) the final assignment.

assigning Core $C$ to TAM 2, because Core $C$ has a lower testing time on the next-narrower TAM (TAM 3) than Core $A$. Hence, we would like to avoid the larger testing time of Core $A$ on TAM 3 in a later iteration. Next, Core $B$ is assigned to TAM 3. TAM 2 is now the minimally loaded TAM; therefore, Core $C$ is assigned to TAM 2. Finally, Core $D$ is assigned to TAM 1. Fig. 2(b) presents the final assignment of cores to TAMs. The testing times on TAMs 1, 2, and 3 are 180, 200, and 200 clock cycles, respectively. The complexity of Core_assign is $\mathcal{O}\left(N^{2}\right)$, where $N$ is the number of cores in the SOC. This can be explained as follows. The While loop in line 9 executes a total of $N$ times since it executes once for each core. For each of these executions, lines 10-12 and lines 13-16 take $\mathcal{O}(N)$ worst case time to select a TAM and a core, respectively. Therefore, the total runtime of Core_assign is $\mathcal{O}\left(N^{2}\right)$. In practice, Core_assign executes two orders of magnitude faster than the ILP model in [10]; hence, a significantly larger number of Core_assign iterations can be executed in the time taken to execute the ILP model.

\section{TAM WIDTH PARTITIONING}

In this section, we describe how the Core_assign heuristic is used to develop an algorithm to quickly reach an intermediate solution to $\mathcal{P}$ PAW and $\mathcal{P}_{\text {NPAW }}$. We also demonstrate how, once an approximate solution for $\mathcal{P}_{\text {NPAW }}$ has been reached, an exact mathematical programming model for $\mathcal{P}_{\text {AW }}$ can be executed to perform a final optimization of the core assignment, thus achieving near-optimal results with little computation time.

\section{A. Fast Algorithm for $\mathcal{P}_{\mathrm{NPAW}}$}

In [10], it was shown that the $\mathcal{P}_{\text {AW }}$ ILP model takes a relatively small time to execute for problem instances of reasonable size. This was exploited by executing the model for each unique TAM width partition and recording the partition and core assignment with the best testing time. Solutions to $\mathcal{P}_{\text {PAW }}$ and $\mathcal{P}_{\text {N PAW }}$ were thus obtained. The number of unique partitions $p_{B}(W)$ for given values of $W$ and $B$ can be estimated using partition theory in combinatorial mathematics [16]. In [16], $p_{B}(W)$ is shown to be approximately $W^{B-1} / B$ ! $(B-1)$ ! for $W \mapsto \infty$. For $B=2, p_{2}(W)=\lfloor W / 2\rfloor$. For $B=3$, the number of partitions can be shown to be $\sum_{i=0}^{\lfloor W / 3\rfloor}[(W-(3 i+1)) / 2\rfloor[10]$. From this formula, $p_{3}(64)=341$. Therefore, the execution time for SOCs having three TAMs is reasonable, even for large $W$. The challenge to effective partition enumeration lies in the fact that for $B \geq 3$, there is no simple and systematic method to enumerate only the unique partitions. In fact, no exact formula is available to calculate the total number of unique partitions for a given value of $W$ and $B$. The value of $p_{B}(W)$ can, thus, only be approximated assuming $W \mapsto \infty$. One way to ensure that only unique partitions are evaluated is to discard, prior to evaluation, each new partition that appears to be a cyclical isomorphism of a previously handled partition. However, the memory requirements for this method and the number of partition comparisons required grow exponentially with $B$ and severely limit the scalability for large $B$. Furthermore, as $B$ increases, the time required to enumerate

unique partitions and evaluate them using ILP increases significantly. The enumeration method in [10] is, therefore, inadequate for industrial SOCs having multiple TAMs.

In this subsection, we use Core_assign to develop a fast method to evaluate width partitions; this effectively addresses the problems inherent to the ILP model and "enumeration-comparison" method described above. The new heuristic employs extensive solution-space pruning, and is, thus, applicable to industrial SOCs having a large number of TAMs. In our experiments with industrial SOCs, we were able to evaluate width partitions and testing times for up to ten TAMs within a few minutes. Test access architectures having more than ten TAMs could also be evaluated, but were found to be less useful for testing time minimization because testing time increases significantly as the relative width of each TAM decreases beyond a threshold.

The TAM optimization algorithm is composed of two parts: a main function Partition_evaluate for partition evaluation and a subroutine Increment that is called to generate partitions. The main function is used to iterate over the number of TAMs designed (to solve $\mathcal{P}_{\text {NPAW }}$ ). For each number of TAMs, the TAM partition is first set to an initial value and the testing time is evaluated using Core_assign as a subroutine. Next, the Increment subroutine is called and a new partition is generated and subsequently evaluated. This continues until all the partitions have been evaluated. Therefore, for each number of TAMs, the $\mathcal{P}$ PAW problem is solved. The lowest testing time recorded during the process is finally output.

The new algorithm Partition_evaluate for problems $\mathcal{P}_{\text {PAW }}$ and $\mathcal{P}_{\text {NPAW }}$ is presented in Fig. 3. Lines 1-3 initialize the values of total TAM width $W$, the set of cores $\mathbf{C}$, and the maximum number of TAMs to be evaluated $B_{\max }$. TAM architectures ranging from 1 to $B_{\max }$ TAMs will be evaluated (line 4 ). In lines 5-8, the loop variable values (TAM widths $w_{1}$ to $w_{B-1}$ ) and the value of lowest total testing time achieved $\mathcal{T}$ are initialized. The done variable that is initialized to FALSE in line 9 will be set to TRUE in Procedure Increment when all width partitions for the $B$ TAMs have been enumerated. In line 10 , Increment is called and the next TAM partition is enumerated; intuitively, Increment works like a $B$-digit counter with upper bounds on the value that each digit can take. In line 11 of Partition_evaluate, the core assignment and total testing time $\mathcal{T}_{\text {new }}$ for the new partition in calculated using Core_assign. If $\mathcal{T}_{\text {new }}$ is less than the lowest testing time achieved $(\mathcal{T})$, the new partition is recorded. After Increment has cycled through all the partitions, the best partition and number of TAMs achieved is output.

Partition_evaluate employs the following three levels of solutionspace pruning.

1) The number of partitions enumerated is significantly limited by the restriction in line 1 of the recursive function Increment. To enumerate partitions of $W$ over $B$ TAMs, Increment dynamically creates $B$ nested loops with loop variables $w_{1} \ldots w_{B}$. The value of each loop variable $w_{1}$ to $w_{B-1}$ is initialized to 1 and is allowed to increment until it reaches an upper bound. The upper bound is based on the value of the TAM width $w_{j}$ currently being incremented, the total number of TAMs $B$, and the sum of widths of the TAMs $w_{1}$ to $w_{j-1}$. Intuitively, this upper bound sums up the values of TAM widths $w_{1}$ to $w_{j-1}$ and divides the sum by the number of TAMs in the list after TAM $j$. Without the upper bound in Line 1, enumeration would be as follows:

$$
\begin{aligned}
\left\{w_{1}+\cdots+w_{B}\right\}= & \{1+\cdots+1+(W-B+1)\}, \\
& \{1+\cdots+2+(W-B)\}, \ldots, \\
& \{(W-B+1)+1+\cdots+1\} .
\end{aligned}
$$

However, a sizeable number of repeated partitions is prevented by establishing an upper bound 


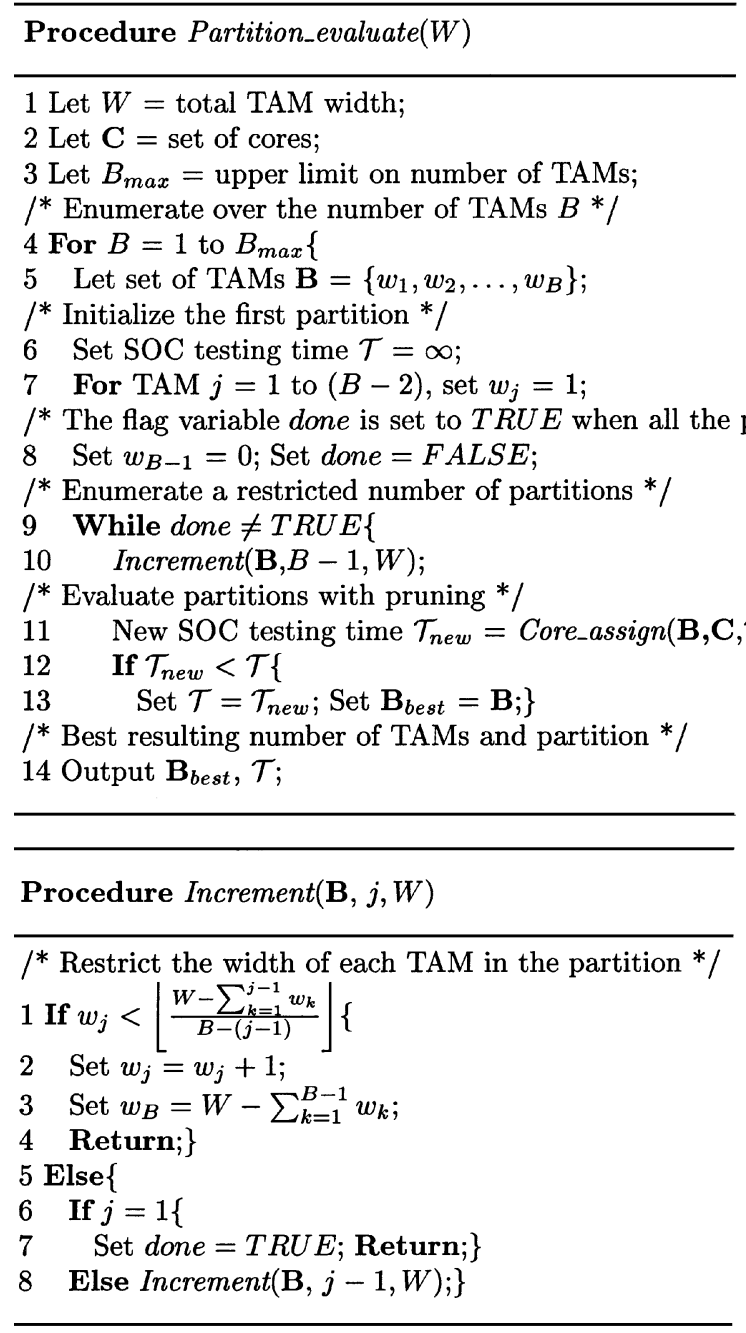

Fig. 3. Fast algorithm for partition evaluation.

$\left\lfloor\left(W-\sum_{k=1}^{j-1} w_{k}\right) /(B-(j-1))\right\rfloor$ on each variable $w_{j}$ during enumeration. For example, for $W=8, B=4$, the first three partitions enumerated are $\{1+1+1+5\},\{1+1+2+4\}$, and $\{1+1+3+3\}$, respectively. If the restriction of line 1 were not present, the repeated partition $1+3+1+3$ would also subsequently be enumerated. However, line 1 establishes an upper bound of 2 on $w_{2}$; thus, partition $1+3+1+3$ is not enumerated.

2) Lines 18-20 in Core_assign terminate the evaluation of any partition for which the testing time $T_{r}$ of any TAM $r$ has already exceeded the best-known value of testing time $\mathcal{T}$ calculated previously. The number of such partitions, whose evaluation can be terminated was found to be large in our experiments; therefore, the execution time of Partition_evaluate is reduced significantly.

3) Partition_evaluate uses heuristic algorithm Core_assign to evaluate partitions. This $\mathcal{O}\left(N^{2}\right)$ algorithm significantly reduces the amount of computation performed.

The complexity of Partition_evaluate can be estimated as follows. The For loop in line 4 will execute a maximum of $W$ times since $B$ can be at most $W$. For each of these executions the number of partitions enumerated is $\mathcal{O}\left(W^{B-1} / B !\right)$. In the worst case, all these partitions will be evaluated to completion. Note that this is the number of partitions enumerated by our algorithm, and not the actual number of unique partitions $p_{B}(W)$, which is given by $W^{B-1} / B !(B-1)$ ! as shown earlier. Now, for each partition evaluated to completion, the
TABLE I

EFFICIENCY OF THE PARTITION_EVALUATE HEURISTIC

\begin{tabular}{c||c|c|c||c|c|c}
\hline \multicolumn{1}{c||}{} & \multicolumn{3}{c||}{$B=6$} & \multicolumn{3}{c}{$B=8$} \\
\cline { 2 - 7 }$W$ & $p_{B}(W)$ & $P_{\text {eval }}$ & $\eta$ & $p_{B}(W)$ & $P_{\text {eval }}$ & $\eta$ \\
\hline \hline 44 & 1909 & 46 & 0.02 & 1571 & 170 & 0.1 \\
48 & 2949 & 46 & 0.02 & 2889 & 48 & 0.02 \\
52 & 4401 & 65 & 0.01 & 5059 & 100 & 0.02 \\
56 & 6374 & 111 & 0.02 & 8499 & 110 & 0.01 \\
60 & 9000 & 278 & 0.03 & 13776 & 172 & 0.01 \\
64 & 12428 & 708 & 0.06 & 21643 & 256 & 0.01 \\
\hline
\end{tabular}

complexity of Core_assign is $\mathcal{O}\left(N^{2}\right)$. Therefore, the complexity of Partition_evaluate can be estimated as $\sum_{B=1}^{W} \mathcal{O}\left(\left(W^{B-1} / B !\right) N^{2}\right)$. While this is not a closed-form expression, it aids in approximating the amount of computation necessary, given values of $W, B$, and $N$.

Table I presents some experimental data on the efficiency of Partition_evaluate for partition-space pruning. The number of possible unique partitions $p_{B}(W)$ [estimated using $p_{B}(W)=$ $\left.W^{B-1} / B !(B-1) !\right]$ is presented for several values of $W$ and $B$. We present results for $W \geq 44$, because the formula $p_{B}(W)=W^{B-1} / B !(B-1)$ ! is accurate only for larger values of $W$. The number of partitions $P_{\text {eval }}$ that are actually evaluated to completion (pruned by Line 1 of the Increment function and by lines 18-20 of Core_assign) is presented for an example SOC p22810 
TABLE II

RESULTS FOR D695: $B=2$ FOR PROBLEM $\mathcal{P}_{\text {PAW }}$

\begin{tabular}{|c|c|c|c|c|c|c|c|c|}
\hline \multirow[b]{2}{*}{$W$} & \multicolumn{3}{|c|}{ Exhaustive enumeration } & \multicolumn{3}{|c|}{ New optimization method } & \multirow[b]{2}{*}{$\begin{array}{l}\Delta \mathcal{T} \\
(\%)\end{array}$} & \multirow[b]{2}{*}{$\begin{array}{l}\text { Ratio } \\
\frac{\mathcal{E}_{\text {new }}}{\mathcal{E}_{\text {old }}}\end{array}$} \\
\hline & $w_{1}+w_{2}$ & $\begin{array}{l}\text { Testing time } \\
\mathcal{T}_{\text {old }} \text { (cycles) } \\
\end{array}$ & $\begin{array}{l}\text { Exec. time } \\
\mathcal{E}_{\text {old }}(\mathrm{sec})\end{array}$ & $w_{1}+w_{2}$ & $\begin{array}{c}\mathcal{T}_{\text {new }} \\
\text { (cycles) }\end{array}$ & $\begin{array}{l}\mathcal{E}_{\text {new }} \\
(\mathrm{sec})\end{array}$ & & \\
\hline 16 & $6+10$ & 45055 & 5 & $8+8$ & 45055 & 1 & +0.00 & 0.2 \\
\hline 24 & $6+18$ & 29501 & 6 & $12+12$ & 34455 & 1 & +16.79 & 0.2 \\
\hline 32 & $11+21$ & 25442 & 6 & $16+16$ & 25828 & 1 & +1.52 & 0.2 \\
\hline 40 & $8+32$ & 21359 & 9 & $20+20$ & 22848 & 1 & +6.97 & 0.1 \\
\hline 48 & $16+32$ & 19938 & 10 & $20+28$ & 22804 & 1 & +14.37 & 0.1 \\
\hline 56 & $19+37$ & 18434 & 12 & $23+33$ & 18940 & 1 & +2.74 & 0.1 \\
\hline 64 & $20+44$ & 18205 & 16 & $32+32$ & 18869 & 1 & +3.65 & 0.1 \\
\hline
\end{tabular}

TABLE III

RESULTS FOR D695: $B=3$ FOR PROBLEM $\mathcal{P}_{\text {PAW }}$

\begin{tabular}{c||c|c|c|c|c|c|c|c}
\hline \multicolumn{1}{l||}{} & \multicolumn{3}{c|}{ Exhaustive enumeration } & \multicolumn{2}{c|}{ New optimization method } & & \\
\cline { 2 - 7 }$W$ & $w_{1}+$ & Testing time & Exec. time & $w_{1}+$ & $\mathcal{T}_{\text {new }}$ & $\mathcal{E}_{\text {new }}$ & $\Delta \mathcal{T}$ & Ratio \\
$W$ & $w_{2}+w_{3}$ & $\mathcal{T}_{\text {old }}$ (cycles) & $\mathcal{E}_{\text {old }}(\mathrm{sec})$ & $w_{2}+w_{3}$ & $($ cycles) & $(\mathrm{sec})$ & $(\%)$ & $\begin{array}{c}\mathcal{E}_{\text {new }} \\
\mathcal{E}_{\text {old }}\end{array}$ \\
\hline \hline 16 & $3+5+8$ & 42568 & 16 & $5+5+6$ & 42952 & 1 & +0.9 & 0.06 \\
24 & $2+5+17$ & 28292 & 42 & $8+8+8$ & 30032 & 1 & +6.15 & 0.02 \\
32 & $4+10+18$ & 21566 & 96 & $8+12+12$ & 24851 & 1 & +15.23 & 0.01 \\
40 & $4+17+19$ & 17901 & 130 & $7+16+17$ & 18448 & 1 & +3.06 & 0.01 \\
48 & $4+19+25$ & 16975 & 182 & $16+16+16$ & 17581 & 1 & +3.57 & 0.01 \\
56 & $5+18+33$ & 13207 & 234 & $17+19+20$ & 15510 & 1 & +17.44 & 0.004 \\
64 & $5+17+42$ & 12941 & 541 & $18+20+26$ & 15442 & 1 & +19.33 & 0.002 \\
\hline
\end{tabular}

from Philips. (The relevant details of this SOC are presented in Section IV.) Finally, the efficiency $\eta$ of our heuristics is calculated using $\eta=P_{\text {eval }} / p_{B}$. Here, $\eta=0.01$ implies that only $1 \%$ of the number of unique partitions are evaluated to completion by Partition_evaluate. We choose SOC p22810 to illustrate the efficiency of our heuristics, because the exhaustive enumeration method [10] was found to be severely inadequate for TAM design for p22810; the method did not complete even for $B=3$. From Table I, it can be seen that Partition_evaluate evaluates on average only $2 \%$ of the unique partitions. Thus, there is a significant reduction in the execution time using this heuristic compared to the exhaustive enumeration method.

\section{B. Final Optimization Step}

Partition_evaluate provides a fast approximation of the optimal values of TAM width partition and testing time. However, this solution can be further improved upon by performing a final optimization step using the ILP model for Problem $\mathcal{P}_{\text {AW }}$ [10]. The best TAM partition obtained from Partition_evaluate is input to the ILP model for $\mathcal{P}_{\text {AW }}$ to calculate the optimal core assignment. This final core assignment and testing time is the final solution of our three-step TAM optimization procedure. Since this final ILP step is performed only once, and since the execution time for a single iteration of the ILP model for $\mathcal{P}_{\text {AW }}$ is relatively small, this results in a near-optimal solution to $\mathcal{P}_{\mathrm{NPAW}}$ in a short execution time. Here, we repeat the ILP model for $\mathcal{P}_{\text {AW }}$ from [10] for reasons of completeness, and to comment on its complexity.

To model $\mathcal{P}_{\text {AW }}$, consider an SOC consisting of $N$ cores and $B$ TAMs of widths $w_{1}, w_{2}, \ldots, w_{B}$. The time taken to test Core $i$ assigned to TAM $j$, given by $T_{i}\left(w_{j}\right)$ clock cycles, is calculated using $D e$ sign_wrapper. We introduce binary variables $x_{i j}$ (where $1 \leq i \leq N$ and $1 \leq j \leq B$ ), which are used to determine the assignment of cores to TAMs in the SOC. Let $x_{i j}$ be a $0-1$ variable defined as follows:

$$
x_{i j}=\left\{\begin{array}{ll}
1, & \text { if Core } i \text { is assigned to TAM } j \\
0, & \text { otherwise }
\end{array} .\right.
$$

The time needed to test all cores on TAM $j$ is given by $\sum_{i=1}^{N} T_{i}\left(w_{j}\right)$. $x_{i j}$. Since all the TAMs can be used simultaneously for testing, the
TABLE IV

NEW RESULTS FOR D695 FOR $\mathcal{P}_{\text {NPAW }}$

\begin{tabular}{c||c|c|c|c|c|c}
\hline \multicolumn{1}{l||}{} & \multicolumn{5}{c|}{ New optimization method } & \\
\cline { 2 - 6 }$W$ & $B$ & $\begin{array}{c}\text { TAM } \\
\text { partition }\end{array}$ & $\begin{array}{c}\mathcal{T}_{\text {new }} \\
\text { (cycles) }\end{array}$ & $\begin{array}{c}\mathcal{E}_{\text {new }} \\
(\mathrm{sec})\end{array}$ & $\begin{array}{c}\Delta \mathcal{T} \\
(\%)\end{array}$ & $\begin{array}{c}\text { Ratio } \\
\frac{\mathcal{E}_{\text {new }}}{\mathcal{E}_{\text {old }}}\end{array}$ \\
\hline \hline 16 & 4 & $3+3+5+5$ & 42644 & 1 & +0.18 & 0.06 \\
24 & 3 & $8+8+8$ & 30032 & 1 & +6.15 & 0.03 \\
32 & 4 & $7+6+9+10$ & 22268 & 1 & +3.26 & 0.02 \\
40 & 3 & $7+16+17$ & 18448 & 1 & +3.06 & 0.01 \\
48 & 5 & $5+2+8+16+17$ & 15300 & 2 & -9.86 & 0.01 \\
56 & 5 & $5+8+11+16+16$ & 12941 & 2 & -2.01 & 0.01 \\
64 & 6 & $5+3+8+12+18+18$ & 12941 & 7 & +0.00 & 0.02 \\
\hline
\end{tabular}

system testing time equals $\max _{1 \leq j \leq B} \sum_{i=1}^{N} T_{i}\left(w_{j}\right) \cdot x_{i j}$. The ILP model for $\mathcal{P}_{\text {AW }}$ can be formulated as follows.

Objective: Minimize testing time $\mathcal{T}$, subject to

1) $\mathcal{T} \geq \sum_{i=1}^{N} T_{i}\left(w_{j}\right) \cdot x_{i j}, 1 \leq j \leq B$, i.e., $\mathcal{T}$ is the maximum testing time on any TAM;

2) $\sum_{j=1}^{B} x_{i j}=1,1 \leq i \leq N$, i.e., every core is assigned to exactly one TAM.

The number of variables and constraints for this model (a measure of its complexity) is given by $N \cdot B$, which is $\mathcal{O}\left(N^{2}\right)$, and $N+B$, which is $\mathcal{O}(N)$, respectively. This ILP model uses the best width partition obtained from Partition_evaluate to optimize the core assignment and obtain a near-optimal TAM architecture in the final step of our optimization methodology.

\section{EXPERIMENTAL RESULTS}

In this section, we present experimental results on our TAM optimization methodology for four example SOCs. The first, d695, is an academic benchmark SOC from Duke University. The other three SOCs, p22810, p34392, and p93791, are from Philips. All four SOCs are part of the ITC'02 SOC test benchmarks initiative [20]. The number (e.g., 93 791) in each SOC name is a measure of its test complexity. We 
TABLE V

RESULTS FOR P22810

\begin{tabular}{|c|c|c|c|c|c|c|c|c|c|}
\hline \multirow[b]{2}{*}{$W$} & \multicolumn{3}{|c|}{ Exhaustive enumeration method } & \multicolumn{4}{|c|}{ New optimization method } & \multirow[b]{2}{*}{$\begin{array}{l}\Delta \mathcal{T} \\
(\%) \\
\end{array}$} & \multirow[b]{2}{*}{$\begin{array}{l}\text { Ratio } \\
\frac{\mathcal{E}_{\text {new }}}{\mathcal{E}_{\text {old }}}\end{array}$} \\
\hline & $w_{1}+w_{2}$ & $\begin{array}{l}\text { Testing time } \\
\mathcal{T}_{\text {old }} \text { (cycles) }\end{array}$ & $\begin{array}{l}\text { Exec. time } \\
\mathcal{E}_{\text {old }}(\mathrm{sec})\end{array}$ & $B$ & $\begin{array}{c}\text { TAM } \\
\text { partition }\end{array}$ & $\begin{array}{c}\mathcal{T}_{\text {new }} \\
\text { (cycles) }\end{array}$ & $\begin{array}{l}\mathcal{E}_{\text {new }} \\
(\mathrm{sec})\end{array}$ & & \\
\hline 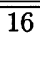 & $6+10$ & 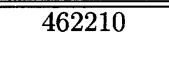 & $\overline{11}$ & 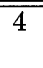 & $\begin{array}{l}1+1+ \\
4+10\end{array}$ & 4468011 & 10 & +1.25 & 0.90 \\
\hline 24 & $8+16$ & 361571 & 24 & 3 & $\begin{array}{c}4+10+ \\
10\end{array}$ & 313607 & 41 & -13.27 & 1.71 \\
\hline 32 & $10+22$ & 312659 & 49 & 4 & $\begin{array}{l}1+10+ \\
10+11\end{array}$ & 246332 & 192 & -21.21 & 3.92 \\
\hline 40 & $10+30$ & 278359 & 60 & 5 & $\begin{array}{c}5+5+10+ \\
10+10\end{array}$ & 232049 & 60 & -16.64 & 1.00 \\
\hline 48 & $10+38$ & 268472 & 84 & 6 & $\begin{array}{c}5+3+10 \\
10+10+10\end{array}$ & 232049 & 15 & -13.57 & 0.18 \\
\hline 56 & $10+46$ & 266800 & 80 & 6 & $\begin{array}{c}5+4+10 \\
10+10+17\end{array}$ & 153990 & 69 & -42.28 & 0.86 \\
\hline 64 & $10+54$ & 260638 & 122 & 6 & $\begin{array}{c}9+8+10+ \\
10+10+17\end{array}$ & 153990 & 138 & -40.92 & 1.31 \\
\hline
\end{tabular}

calculate the SOC test complexity number using the formula presented in [20].

The experimental results presented in this paper were obtained at Duke University using a Sun Ultra 10 with a $333-\mathrm{MHz}$ processor and $256 \mathrm{MB}$ of memory. All SOC testing times in this section are expressed in clock cycles.

\section{A. Results for SOC $d 695$}

In this subsection, we present experimental results for SOC d695. SOC d695 consists of two ISCAS' 85 and eight ISCAS' 89 benchmark circuits [10]. Tables II and III compare the results obtained using the method in [10] with the results of the new TAM optimization method for d695 for Problem $\mathcal{P}_{\text {PAW }}$ for $B=2$ and $B=3$. The testing times $\mathcal{T}_{\text {new }}$ of the new method are comparable to the testing times $\mathcal{T}_{\text {old }}$ in [10]. However, the CPU times $\mathcal{E}_{\text {new }}$ of the new method are in most cases significantly less than the CPU times $\mathcal{E}_{\text {old }}$ using [10] for larger values of $W$. The percentage change in testing time using the new method is calculated using the formula $\Delta \mathcal{T}(\%)=\left(\left(\mathcal{T}_{\text {new }}-\mathcal{T}_{\text {old }}\right) / \mathcal{T}_{\text {old }}\right) \times 100$.

While the methods of [10] are limited to $B \leq 3$ due to high computation cost, using the heuristic proposed in this paper, we are now able to search a much larger solution-space. We can now design test architectures having a larger number of TAMs that are more efficient than limited-size architectures. Table IV presents the results obtained with the new TAM optimization methodology for d695 over a larger number of TAMs (problem $\mathcal{P}_{\text {NPAW }}, B \leq 10$ ). The testing times obtained using the new optimization technique are better than or equal to the best testing times in [10] for larger values of $W$. For $W \leq 40$, the new testing times are on average only $3 \%$ larger than those reported in [10]. We improve upon the testing times compared to [10] for larger values of $W$ because the exhaustive enumeration approach of [10] simply could not compute test architectures having more than three TAMs for these values of $W$. Since, in [10], the exhaustive enumeration method did not terminate within a reasonable CPU time for $B>3$, the ratio $\mathcal{E}_{\text {new }} / \mathcal{E}_{\text {old }}$ reported in Table IV is obtained using the value of $\mathcal{E}_{\text {old }}$ for $B=3$. There is an improvement of two orders of magnitude in the CPU times in all cases.

We have also investigated the increase in the computation time (compared to the times presented in Table IV), if the final optimization step is performed for the partitions obtained from Partition_evaluate for each value of $B$. For the different values of $W$ for d695, the new computation times are as follows. Each of the following tuples has the format ( $W, \mathcal{T}_{1}, \mathcal{T}_{2}$ ), where $\mathcal{T}_{1}$ represents the original computation time (from Table IV) for TAM width $W$ and $\mathcal{T}_{2}$ represents the new computation time if the final optimization step is performed for each value of $B$ : $(16,1,2),(24,1,2),(32,1,3),(40,1,4),(48,2,4),(56,2,9),(64,7,14)$. These results show that the computation time is small even if the time needed for the final optimization step is taken into account.

\section{B. Results for SOC p22810}

SOC p22810 contains 28 cores. Of these, six are memory cores and 22 are scan-testable logic cores [20]. Table V compares the results for p22810 obtained using the method in [10] for $B=2$ with the results for a larger number of TAMs $(1 \leq B \leq 10)$ using the new optimization method. The exhaustive enumeration method [10] did not run to completion for $B=3$, even after two days of execution. For $W \geq 24$, the testing times obtained using the new optimization technique are on average $25 \%$ lower than those obtained using [10]. This is because using Partition_evaluate, we were able to partition $W$ among a larger number of TAMs than was possible using exhaustive enumeration. The new CPU times are comparable to the CPU times of the exhaustive enumeration method. The final optimization step took between 10-20 seconds to complete in all cases. This was because the ILP models for this SOC were particularly intractable. This also explains why the exhaustive enumeration method from [10] did not terminate within a reasonable CPU time for $B=3$ for $\mathrm{p} 22810$.

For $\mathcal{P}_{\text {NPAW }}$ and $W=16$, the Partition_evaluate heuristic returns a partition of $1+1+4+10$. This yields a testing time of 468011 cycles after the final optimization step (Table V). However, a lower testing time of 462210 cycles is actually achievable using only two TAMs. This is because Partition_evaluate returns a lower testing time for four TAMs than for two TAMs before the final optimization step. This anomalous behavior of our algorithm is due to the fact that Partition_evaluate uses heuristics to quickly approximate the final result. Therefore, the partition that actually provides the lowest testing time after final (exact) optimization might not be returned by Partition_evaluate. Similarly, for $W=64$, Partition_evaluate obtains a partition of $13+10+10+10+21$ (five TAMs), which gives a lower (heuristic) testing time than does the partition obtained for $W=56: 5+4+10+10+10+17$ (six TAMs). However, after the final optimization step, the partition for $W=56$ is able to achieve the same testing time as that for $W=64$.

In Fig. 4, we illustrate the effectiveness of the new heuristic method in exploring a larger solution-space than was possible using the exhaustive enumeration method of [10]. Fig. 4(a) presents the TAM design for SOC p22810 for $W=64$ using the methods in [10] for $B=2$ (the exhaustive enumeration method did not complete for $B=3$ ). Two TAMs provide a testing time of 260638 cycles. Fig. 4(b) illustrates the 


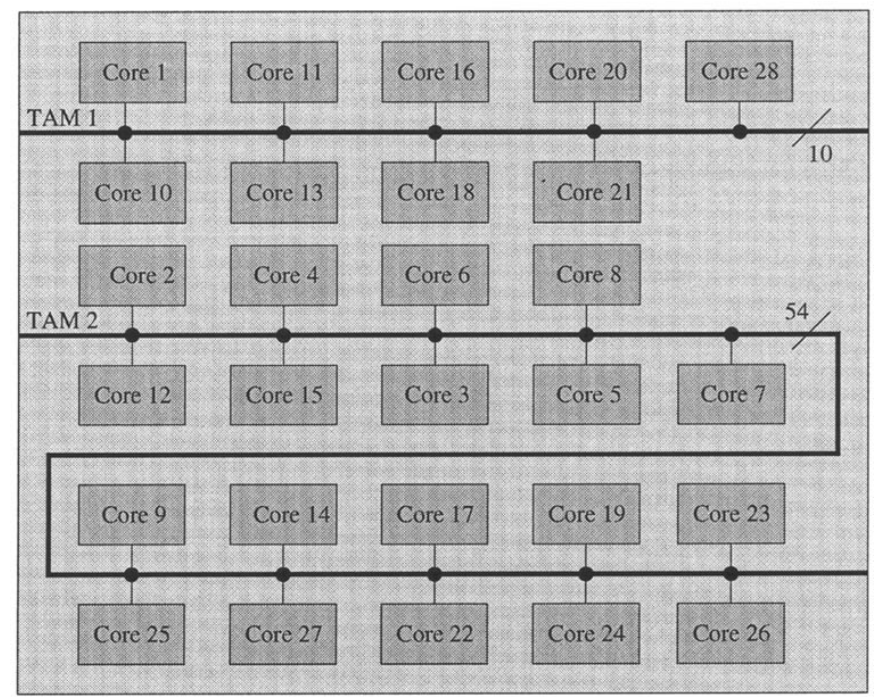

Testing time $=260638$ cycles

(a)

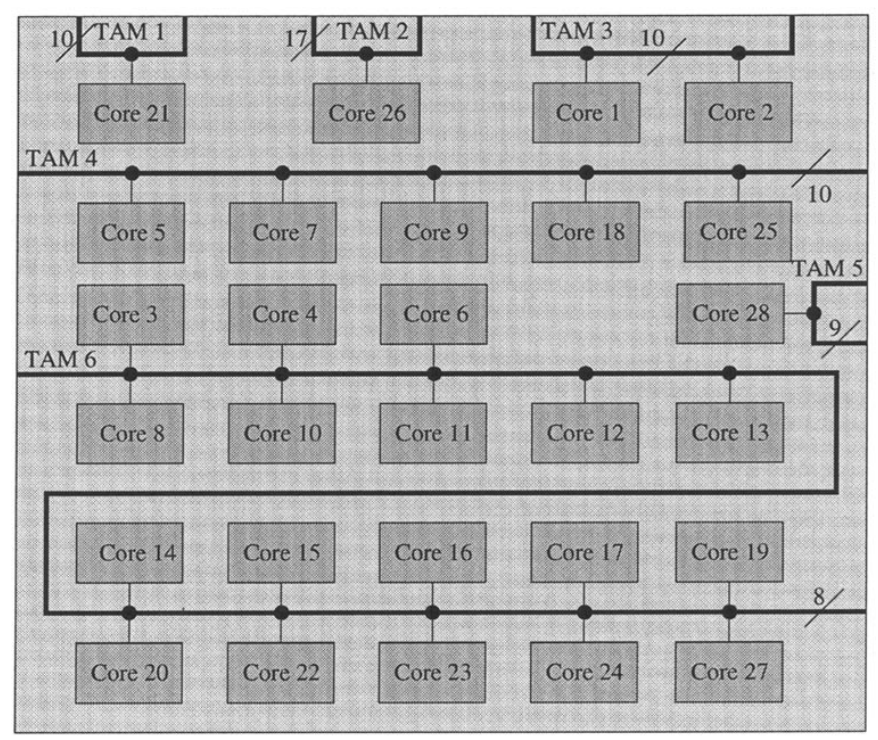

Testing time $=153990$ cycles

(b)

Fig. 4. Illustration of TAM design for p22810. (a) Best two-TAM architecture using exhaustive enumeration method with $W=64$. (b) Best six-TAM architecture using new heuristic method.

TAM architecture obtained using the new heuristic method. Using the new method, it is possible to design a six-TAM system that provides a testing time of 153990 cycles, a saving of $40.92 \%$ over [10].

\section{Results for SOC $p 34392$}

SOC p34392 contains 19 cores. Of these, 15 are memory cores and four are scan-testable logic cores [20]. Table VI compares the results obtained by the exhaustive enumeration method with the results obtained by the new optimization method for p34392. For $B=4$, the exhaustive enumeration method of [10] did not provide a solution even after two days of CPU time.

For $W \leq 32$, the testing times obtained using the new optimization technique are on average $15 \%$ higher than those obtained using the exhaustive enumeration method. For $W \geq 40$, we reach the optimum testing time of 544579 cycles. The testing time of this SOC does not decrease beyond 544579 cycles as $W$ is increased beyond 40 and $B$ is increased beyond three. This is because the testing time for Core 18 in p34392 reaches a minimum value of 544579 cycles when the width of the TAM to which it is assigned reaches 10 bits. Thus, our method achieves the theoretical lower bound on testing time for this SOC. The new CPU times are on average between one and two orders of magnitude less than the CPU times of the exhaustive enumeration method. This is because the individual $\mathcal{P}_{\text {AW }}$ ILP models for p34392 took particularly long to solve. This significantly affected the CPU time of the exhaustive enumeration method for $\mathcal{P}_{\text {PAW }}$ and $\mathcal{P}_{\text {NPAW }}$.

\section{Results for SOC p93791}

SOC p93791 contains 32 cores [10]. Of these 32 cores, 18 are memory cores and 14 are scan-testable logic cores [20]. Table VII compares the exhaustive enumeration results for p93791 with the new results. Note that the new optimization method results in optimum testing times for several values of $W$. Here, too, we did not achieve a solution with the exhaustive enumeration method after two days of execution for $B=4$. The testing times obtained using the new optimization technique are comparable to the best testing times using the exhaustive enumeration method for all values of $W$, and equal to the results in [10] for $W=48$ and 56. The testing times are on average 3\% larger than those reported in [10]. The CPU times of the new Partition_evaluate algorithm are between two and three orders of magnitude smaller than the CPU times of the exhaustive enumeration method. This is because Partition_evaluate is able to effectively prune the solution space by halting evaluation of unnecessary partitions, for which the testing time of a TAM exceeds the previous minimum value for the SOC. For example, of the 341 unique partitions for $W=64$ and $B=3$, only 23 were evaluated to completion. Therefore, the new heuristic algorithm Core_assign makes it possible to evaluate TAM architectures for industrial SOCs having multiple TAMs, which was not feasible using the methods in [10].

\section{CONCLUSION}

We have presented a new efficient technique for optimization of the TAM architecture for industrial SOCs. The general TAM optimization problem has been formulated as a progression of four problems. The first problem $\mathcal{P}_{\mathbf{W}}$ relating to wrapper design is solved using an efficient algorithm presented in an earlier paper. For the second problem $\mathcal{P}_{\text {Aw }}$, that of core assignment among TAMs of fixed widths, we have presented an efficient procedure called Core_assign that executes significantly faster than an ILP model for the same problem presented earlier, thereby leading to the rapid estimation of the quality of a particular TAM partition. The third and fourth problems in the progression $\mathcal{P}_{\text {PAW }}$ and $\mathcal{P}_{\text {NPAW }}$ relate to determining a partition of TAM width and an effective number of TAMs for the SOC, such that testing time is minimized. These two problems have been solved using a new heuristic procedure called Partition_evaluate that quickly reaches within the neighborhood of the optimal solution to $\mathcal{P}_{\text {PAW }}$ and $\mathcal{P}_{\text {NPAW }}$. Partition_evaluate uses extensive solution-space pruning to identify an effective TAM partition for the SOC. Core_assign and Partition_evaluate together facilitate the quick identification of a high-quality TAM partition for the SOC. Finally, the existing ILP model for $\mathcal{P}_{\text {AW }}$ is used to optimize the core assignment and testing time for the width partition produced by Partition_evaluate. Experimental results for several industrial SOCs demonstrate that TAM optimization can be effectively carried out in significantly less time than exact methods based on ILP and exhaustive enumeration presented earlier. This facilitates the design of a larger number of TAMs and, in certain cases, lower testing times, than was possible using the exact methods proposed earlier. 
TABLE VI

RESULTS FOR P34392

\begin{tabular}{|c|c|c|c|c|c|c|c|c|c|}
\hline \multirow[b]{2}{*}{$W$} & \multicolumn{3}{|c|}{ Exhaustive enumeration method } & \multicolumn{4}{|c|}{ New optimization method } & \multirow[b]{2}{*}{$\begin{array}{l}\Delta \mathcal{T} \\
(\%)\end{array}$} & \multirow[b]{2}{*}{$\begin{array}{l}\text { Ratio } \\
\frac{\mathcal{E}_{\text {new }}}{\mathcal{E}_{\text {old }}}\end{array}$} \\
\hline & $\begin{array}{c}w_{1}+ \\
w_{2}+w_{3}\end{array}$ & $\begin{array}{l}\text { Testing time } \\
\mathcal{T}_{\text {old }} \text { (cycles) } \\
\end{array}$ & $\begin{array}{l}\text { Exec. time } \\
\mathcal{E}_{\text {old }}(\mathrm{sec})\end{array}$ & $B$ & $\begin{array}{c}\text { TAM } \\
\text { partition }\end{array}$ & $\begin{array}{c}\mathcal{T}_{\text {new }} \\
\text { (cycles) }\end{array}$ & $\begin{array}{l}\mathcal{E}_{\text {new }} \\
(\mathrm{sec}) \\
\end{array}$ & & \\
\hline$\overline{16}$ & $=1+7+8$ & 998733 & 222 & 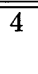 & $\begin{array}{c}3+3+ \\
5+5\end{array}$ & $\overline{1033210}$ & $\overline{27}$ & +3.45 & 0.12 \\
\hline 24 & $9+7+8$ & 720858 & 325 & 4 & $\begin{array}{c}5+5+ \\
6+8\end{array}$ & 882182 & 27 & +22.38 & 0.08 \\
\hline 32 & $\begin{array}{c}17+5+ \\
10\end{array}$ & 591027 & 1576 & 5 & $\begin{array}{c}5+4+ \\
6+8+9\end{array}$ & 663193 & 51 & +12.21 & 0.03 \\
\hline 40 & $\begin{array}{c}9+10+ \\
21\end{array}$ & 544579 & 1081 & 4 & $\begin{array}{l}3+7+ \\
15+15\end{array}$ & 544579 & 39 & +0.00 & 0.04 \\
\hline 48 & $\begin{array}{c}9+10+ \\
29\end{array}$ & 544579 & 6198 & 5 & $\begin{array}{c}5+3+8+ \\
15+17\end{array}$ & 544579 & 205 & +0.00 & 0.03 \\
\hline 56 & $\begin{array}{c}9+10+ \\
37\end{array}$ & 544579 & 11331 & 6 & $\begin{array}{l}5+3+5+ \\
8+15+20\end{array}$ & 544579 & 109 & +0.00 & 0.01 \\
\hline 64 & $\begin{array}{c}17+15+ \\
32\end{array}$ & 544579 & 1125 & 6 & $\begin{array}{l}5+3+5+ \\
8+15+28 \\
\end{array}$ & 544579 & 288 & +0.00 & 0.26 \\
\hline
\end{tabular}

TABLE VII

RESULTS FOR P93791

\begin{tabular}{|c|c|c|c|c|c|c|c|c|c|}
\hline \multirow[b]{2}{*}{$W$} & \multicolumn{3}{|c|}{ Exhaustive enumeration method } & \multicolumn{4}{|c|}{ New optimization method } & \multirow[b]{2}{*}{$\begin{array}{l}\Delta \mathcal{T} \\
(\%)\end{array}$} & \multirow[b]{2}{*}{$\begin{array}{l}\text { Ratio } \\
\frac{\mathcal{E}_{\text {new }}}{\mathcal{E}_{\text {old }}}\end{array}$} \\
\hline & $\begin{array}{c}w_{1}+ \\
w_{2}+w_{3} \\
\end{array}$ & $\begin{array}{l}\text { Testing time } \\
\mathcal{T}_{\text {old }} \text { (cycles) } \\
\end{array}$ & $\begin{array}{l}\text { Exec. time } \\
\mathcal{E}_{\text {old }}(\mathrm{sec}) \\
\end{array}$ & $B$ & $\begin{array}{c}\text { TAM } \\
\text { partition }\end{array}$ & $\begin{array}{c}\mathcal{T}_{\text {new }} \\
\text { (cycles) }\end{array}$ & $\begin{array}{l}\mathcal{E}_{\text {new }} \\
(\mathrm{sec}) \\
\end{array}$ & & \\
\hline$\overline{16}$ & $5+3+8$ & 1771720 & 23 & $\overline{3}$ & $\overline{5+5+6}$ & 1786200 & 2 & +0.82 & 0.09 \\
\hline 24 & $7+8+9$ & 1187990 & 34 & 3 & $8+8+8$ & 1209420 & 3 & +1.80 & 0.09 \\
\hline 32 & $4+5+23$ & 887751 & 102 & 2 & $9+23$ & 894342 & 1 & +0.74 & 0.009 \\
\hline 40 & $6+12+23$ & 698583 & 156 & 3 & $6+10+24$ & 741965 & 1 & +4.60 & 0.006 \\
\hline 48 & $9+16+23$ & 599373 & 194 & 3 & $9+16+23$ & 599373 & 3 & +0.00 & 0.02 \\
\hline 56 & $10+23+23$ & 514688 & 224 & 3 & $10+23+23$ & 514688 & 3 & +0.00 & 0.01 \\
\hline 64 & $18+23+23$ & 460328 & 631 & 3 & $15+23+26$ & 473997 & 2 & +2.96 & 0.003 \\
\hline
\end{tabular}

The heuristic methods presented in this paper can sometimes exhibit anomalies. For example, the width partition and number of TAMs returned by Partition_evaluate do not always provide the lowest testing time after the final (exact) optimization step is performed. However, by selecting several different partitions for final optimization, e.g., performing the final optimization step on the best width partition for each value of $B, 1 \leq B \leq 10$, and then choosing the partition that returns the lowest testing time, potentially anomalous behavior can be avoided. A careful investigation of the heuristic choices driving the selection of partitions for the final optimization step is, therefore, warranted.

\section{ACKNOWLEDGMENT}

The authors thank H. D. Hollman for his help with partition theory. They are grateful to G. Francis, H. van Herten, and E. Waterlander for their help with providing data for the Philips SOCs, and to B. Vermeulen, H. Vranken, and G. Francis for their comments on an earlier version of this paper.

\section{REFERENCES}

[1] J. Aerts and E. J. Marinissen, "Scan chain design for test time reduction in core-based IC's," in Proc. Int. Test Conf., 1998, pp. 448-457.

[2] M. Berkelaar. Lpsolve 3.0. Eindhoven Univ. Technol., Eindhoven, The Netherlands. [Online]. Available: ftp://ftp.ics.ele.tue.nl/pub/lp_solve

[3] P. Brucker, Scheduling Algorithms, 3rd ed. Berlin, Germany: Springer-Verlag, 2001.

[4] K. Chakrabarty, "Design of system-on-a-chip test access architectures under place-and-route and power constraints," in Proc. Design Automation Conf., 2000, pp. 432-437.

[5] - "Optimal test access architectures for system-on-a-chip," ACM Trans. Design Automation Electron. Syst., vol. 6, pp. 26-49, Jan. 2001.
[6] M. R. Garey and D. S. Johnson, Computers and Intractability: A Guide to the Theory of NP-Completeness. San Francisco, CA: Freeman, 1979.

[7] J. Hromkovic, Algorithmics for Hard Problems. Berlin, Germany: Springer-Verlag, 2001.

[8] Y. Huang, W.-T. Cheng, C.-C. Tsai, N. Mukherjee, O. Samman, Y. Zaidan, and S. M. Reddy, "Resource allocation and test scheduling for concurrent test of core-based SOC design," in Proc. Asian Test Symp., 2001, pp. 265-270.

[9] - " "On concurrent test of core-based SOC design," J. Electron. Testing: Theory Applicat., vol. 18, pp. 401-414, 2002.

[10] V. Iyengar, K. Chakrabarty, and E. J. Marinissen, "Test wrapper and test access mechanism co-optimization for system-on-chip," J. Electron. Testing: Theory Applicat., vol. 18, pp. 213-230, 2002.

[11] V. Iyengar and K. Chakrabarty, "Test bus sizing for system-on-a-chip," IEEE Trans. Comput., vol. 51, pp. 449-459, May 2002.

[12] V. Iyengar, K. Chakrabarty, and E. J. Marinissen, "On using rectangle packing for SOC wrapper/TAM co-optimization," in Proc. VLSI Test Symp., 2002, pp. 253-258.

[13] S. Koranne, "On test scheduling for core-based SOC's," in Proc. Int. Conf. VLSI Design, 2002, pp. 505-510.

[14] E. Larsson and Z. Peng, "An integrated system-on-chip test framework," in Proc. Design Automation Test Eur., 2001, pp. 138-144.

[15] _ _ "An integrated framework for the design and optimization of SOC test solutions," J. Electron. Testing: Theory Applicat., vol. 18, pp. $385-400,2002$

[16] J. H. van Lint and R. M. Wilson, A Course in Combinatorics. Cambridge, U.K.: Cambridge Univ. Press, 1992.

[17] E. J. Marinissen et al., "A structured and scalable mechanism for test access to embedded reusable cores," in Proc. Int. Test Conf., 1998, pp. 284-293.

[18] E. J. Marinissen, S. K. Goel, and M. Lousberg, "Wrapper design for embedded core test," in Proc. Int. Test Conf., 2000, pp. 911-920.

[19] M. Nourani and C. Papachristou, "An ILP formulation to optimize test access mechanism in system-on-chip testing," in Proc. Int. Test Conf., 2000, pp. 902-910. 
[20] E. J. Marinissen, V. Iyengar, and K. Chakrabarty, "A set of benchmarks for modular testing of SOC's," in Proc. Int. Test Conf., 2002, pp. 519-528.

[21] IEEE P1500 Standard for Embedded Core Test [Online]. Available: http://grouper.ieee.org/groups/1500

[22] P. Varma and S. Bhatia, "A structured test re-use methodology for corebased system chips," in Proc. Int. Test Conf., 1998, pp. 294-302.

[23] Y. Zorian, E. J. Marinissen, and S. Dey, "Testing embedded-core-based system chips," IEEE Trans. Comput., vol. 32, pp. 52-60, June 1999.

\section{Some Conditions Under Which Hierarchical Verification is $O(N)$}

\author{
Louis K. Scheffer
}

\begin{abstract}
Before manufacturing, each chip design must be inspected for possible errors. For example, design rule checking is used to check for adherence to the physical manufacturing rules, and static timing analysis is used to verify that the design has adequate performance. Although modern chip designs are invariably specified hierarchically, verification algorithms can treat this hierarchy in different ways. The most straightforward way to verify a hierarchical design is to expand (or flatten) the hierarchy, then do the test. Since most verification algorithms involve at least sorting the data, such algorithms are at least $O(N \log N)$, where $N$ is the size of the flattened hierarchy.

Alternatively, a verification tool can try to use the hierarchy rather than flattening it. One form of hierarchical verification involves verifying each cell, generating a model (also called an abstract) for each cell, and then checking all interactions between cells by using their abstracts. Under some conditions, this may be more efficient than flattening the hierarchy and then verifying. In particular, if the size of the abstract is $O\left(n^{a}\right)$ for a cell containing $n$ primitives, and the time to verify a cell and generate the abstract is $O\left(n^{b}\right)$, then the complete verification of all levels of the hierarchy is $O(N)$, provided $a b<1$.
\end{abstract}

Index Terms-Algorithms, complexity theory, computational gemotry, computation time, design automation, hierarchical systems, software performance.

\section{INTRODUCTION}

Before manufacturing, designers need to check various features of a design, both electrical and physical, to verify that the chip will work properly and can be manufactured successfully. Most chips are designed hierarchically, where cells are defined once and used one or more times. A verification program such as design rule checking (DRC) can use this hierarchy in many different ways, with different performance and complexity tradeoffs. Let $H$ be the size of the hierarchical design, and $N$ the size of the same design when flattened. Usually $H$ is much smaller than $N$, though in the worst case (each definition is used only once) it can be slightly bigger.

The simplest approach to verifying a hierarchical design is to ignore hierarchy, by flattening the design first. Clearly this will be at least $O(N)$ since all data must be inspected. Most rule checking algorithms,

Manuscript received March 8, 2002; revised August 25, 2002. This work was supported by Hewlett-Packard from 1977 to 1981. This paper was recommended by Associate Editor Chung-Kuan Cheng.

The author is with Cadence Design Systems, San Jose, CA 95134 USA (e-mail: lou@cadence.com).

Digital Object Identifier 10.1109/TCAD.2003.810740 such as DRC, must at least sort the data, or insert it in trees, leading to algorithms that are $O(N \log N)$ or worse, at least in theory. ${ }^{1}$

A different approach is to take advantage of the design hierarchy instead of flattening it out of existence. Hierarchical verification is defined as the verification that takes a hierarchical description as input and attempts to use the hierarchy to improve the verification process. It should find the same errors, or lack of errors, as the corresponding flat verification.

Though more complex, hierarchical verification has potential advantages:

- potentially better run times: if the checking process is worse than linear, it is better to check many smaller instances than one big instance [10], if the interaction check is not too costly (this advantage holds even if no cells are reused).

- smaller peak memory requirements;

- efficiency from structure: even a flat algorithm can benefit by using the underlying hierarchy to speed geometric searches [5];

- efficiency from repetition: repeated cells are only checked once [11];

- better error reporting: errors in repeated cells are only reported once [11];

- different design rules can be used for different parts of the design [8].

This paper covers only the first advantage, although the others are also very useful in practice.

Since $H$ is usually much smaller than $N$, is there an algorithm that is linear, or at least polynomial, in $H$ ? For one of the main tasks of interest (DRC), it is extremely unlikely this can be guaranteed since hierarchical DRC is nondeterministic-polynomial (NP)-complete [2], [3]. This is demonstrated by transforming any integer knapsack problem into a hierarchical DRC problem in linear time. [This construction creates a design where $N$ is exponentially bigger than $H$, so NP-completeness in $H$ is not contradicted by the existence of $O(N \log N)$ or $O(N)$ algorithms.] See the appendix for a sketch of this proof.

Considerable work in this field is only available in the form of theses [1], [10], technical reports [2], [11], and older conferences [3], [5], [9] not yet accessible to online search systems. This paper puts some of the results and discussion in the machine searchable literature.

\section{HIERARCHICAL ANALYSIS}

One possible way to do hierarchical checking is as follows. First, check all the leaf cells. For each cell build an "abstract," a (hopefully) simpler version of the cell that only contains the features that are needed for checking cell interactions. For example, in timing analysis the abstract might contain only the combinatorial logic attached to the pins. Any path completely internal to the cell (such as from one flip-flop to another in the same cell) can be omitted.

Next, verify the interactions between the cells one hierarchical level at a time. First, verify the cells that contain the leaf cells, by substituting in their abstracts, then run the flat verification algorithm on the resulting data. Then prepare the abstract for the next higher level, and repeat the process until the cell at the top of the hierarchy is verified. At this point, all cells and interactions have been checked.

This process is illustrated in Fig. 1. The objective is to check a layout for spacing errors. Fig. 1(a) shows one layer of a cell. The cell itself has no errors. Fig. 1(b) shows a design that includes two copies of the cell in Fig. 1(a) and two polygons. There are two errors caused by interac-

${ }^{1}$ Some DRC programs index into tables using physical coordinates. For the size of $N$ practical for chip design, this can make up to $\log N$ decisions in a single operation, leading to algorithms that are $O(N)$ in practice. 